\title{
Complication Rates of Hemiarthroplasty Conversion to Total Hip Arthroplasty Versus Primary Total Hip Arthroplasty
}

\author{
Sione A. Ofa, BS', Austin J. Ross, BS', Bailey J. Ross, BA', Oliva C. Lee, MD'2, William F. Sherman, MD, MBA' a \\ 1 Department of Orthopaedic Surgery, Tulane University School of Medicine, 2 Department of Orthopaedic Surgery, Tulane University School of \\ Medicine; Department of Orthopaedic Surgery, Louisiana State University School of Medicine and Southeast Louisiana Veterans Health \\ Keywords: primary total hip arthroplasty, total hip arthroplasty, conversation, hemiarthroplasty, hip fracture, hip
}

https://doi.org/10.52965/001c.25539

\section{Orthopedic Reviews}

Vol. 13, Issue 1, 2021

\section{Background}

One of the most common surgical options for treatment of a femoral neck fracture is hemiarthroplasty (HA). However, progression of arthritis or pain can necessitate conversion to total hip arthroplasty (THA). While conversion to a THA is a viable option, it does carry multiple risks. The purpose of this study was to identify whether performing conversion from HA to THA carries an increased risk of post-operative joint complications when compared to elective THA.

\section{Methods}

An administrative claims database was queried to identify patients who underwent conversion from a HA to a THA. Incidences of prosthetic dislocation, prosthetic joint infection (PJI), periprosthetic fracture, aseptic loosening, and revision were collected and compared to elective primary THA with multivariable logistic regression.

\section{Results}

Patients undergoing conversion THA had significantly higher risks of all joint complications examined at both 1 and 2 years after surgery. These included prosthetic dislocation (1-year: OR 2.95; 2 years: OR 3.77), PJI (1-year: OR 1.38; 2 years: OR 2.13), periprosthetic fracture (1-year: OR 2.95; 2 years: OR 3.75), aseptic loosening (1-year: OR 6.86; 2 years: OR 7.70), and revision (1-year: OR 3.65; 2 years: OR 6.73).

\section{Conclusion}

Performing conversion arthroplasty from HA to THA is associated with an increased risk of multiple joint complications in both the short and mid-term follow-up period. Surgeons should consider these complications when indicating HA for femoral neck fractures and elective conversion arthroplasty.

\section{INTRODUCTION}

Total hip arthroplasty (THA) is a highly successful surgery for the management of hip pathology that yields excellent short-term and long-term outcomes. ${ }^{1}$ Of the estimated 370,000 THAs performed annually in the United States, over $90 \%$ are performed electively to manage hip osteoarthritis. ${ }^{2}$ Total hip arthroplasty is also a viable option for managing femoral neck fractures. However, performing THA for frac- ture indications carries an increased risk of perioperative and postoperative complications. ${ }^{3-5}$ Potential postoperative complications after THA include pain, prosthetic dislocation, fracture, infection, and abductor insufficiency. 6,7

Hemiarthroplasty (HA) is one of the most common treatments for femoral neck fractures. ${ }^{8}$ HA has been shown to yield better results than internal fixation for displaced femoral neck fractures in the elderly as it permits full weight-bearing immediately after surgery without the risk of complications such as avascular necrosis. ${ }^{9}$ However, HA

\footnotetext{
a Corresponding author:

William F. Sherman, MD, MBA

1430 Tulane Avenue

New Orleans, LA 70112

Email: swilliam1@tulane.edu

Tel: 504-982-0252
} 
has been linked to higher reoperation rates and worse functional outcomes compared to THA in community ambulators and active patients. ${ }^{4,10,11}$ Common indications for reoperation after HA include aseptic femoral loosening, periprosthetic fractures, and development of acetabular arthritis. $^{12}$

With the development of acetabular arthritis, conversion of HA to THA is commonly performed for pain relief to remove the articular bearing against the metal prosthetic femoral head. ${ }^{13-15}$ Compared to primary THA, HA conversion to THA has been associated with higher rates of reoperations and complications. ${ }^{16-19}$ Studies demonstrating matched cohorts have involved conversions done for all failed hip fractures, including those receiving cephalomedullary nails, percutaneous screw fixation, and sliding hip screws. However, there is little data comparing large, matched cohorts to evaluate the complication rates to evaluate HA conversion to elective THA. It was hypothesized that HA conversion would be associated with an increased risk of adverse outcomes despite the surgical procedure primarily addressing the acetabulum during a HA conversion.

\section{METHODS AND MATERIALS}

\section{DATA SOURCE}

A retrospective cohort study was designed in which patient records were queried from PearlDiver (PearlDiver Inc. Fort Wayne, IN), a commercially available administrative claims database. This study used data from the "MHip" dataset provided within PearlDiver, which contains approximately 1 million patients from 2010 through Q1 of 2020. Primary and secondary outcomes were defined using International Classification of Disease, Ninth Revision and Tenth Revision (ICD-9 and ICD-10) codes, as well as Current Procedural Terminology (CPT) codes. Institutional Review Board exemption was granted through Tulane University Human Research and Protection Program as the provided data were deidentified and compliant with the Health Insurance Portability and Accountability Act.

\section{PATIENT SELECTION}

Patients aged 65 and older were included. Using CPT codes, patient records were selected and separated into either the "conversion" cohort if they had undergone HA (CPT-27236 and CPT-27125), then conversion of a previous HA to THA with or without allograft (CPT-27132), or a "primary THA" cohort if they had undergone a primary THA (CPT-27130). The code CPT-27236 is for open treatment of femoral neck fracture with internal fixation or prosthesis. Given the specified age to only include patients over 65 , the likelihood of capturing failed open reductions with internal fixation in the conversion cohort was minimized. Becket al demonstrated that $34 \%$ percent of femoral neck fractures that receive HA are incorrectly coded as CPT-27125, which is for elective HA. ${ }^{20}$ Both CPT-27236 and CPT- 27215 were included to catch all HA conversions to THA, whether the HA was for a femoral neck fracture or other indications.

Cohorts were then matched based on gender, region, and Charlson Comorbidity Index (CCI), to create similar popu- lations. To ensure a minimum 2-year follow-up in the database for all included patients in both cohorts, only patients who underwent primary THA or conversion of previous hip surgery between 2010 through the end of 2018 were included. Exclusion criteria for both cohorts included arthroplasty for pathologic fractures or arthroplasty procedures that were miscoded as revision arthroplasty. PPatients were also excluded from the conversion cohort if they had undergone closed reduction with percutaneous pinning (CRPP) for a proximal femur fracture. The ICD codes that defined the different cohorts are provided in Appendix Table A1.

\section{OUTCOMES}

This study's primary outcomes included examining prosthetic joint infection rates (PJI), prosthetic joint dislocation, periprosthetic fracture, aseptic loosening, and revisions at both 1 and 2 years post-surgery. PJI was defined by procedural codes that indicated a surgical intervention for a deep joint infection and excluded any superficial complications such as stitch abscesses or wound complications. In addition to looking at joint complication rates, each cohort was queried for basic demographic and clinical information such as sex, BMI, and comorbidities. Comorbidities examined included tobacco use, rheumatoid arthritis, liver disease, congestive heart failure (CHF), coronary artery disease (CAD), ischemic heart disease (IHD), pulmonary heart disease (PHD), chronic obstructive pulmonary disease (COPD), chronic kidney disease (CKD), dementia, diabetes mellitus, hypertension, and peripheral vascular disease (PVD).

\section{STATISTICAL ANALYSIS}

All data analyses were performed using the $\mathrm{R}$ statistical software (R Project for Statistical Computing, Vienna, Austria) integrated within PearlDiver with an $\alpha$ level set to 0.05. Demographic and clinical characteristics were compared using chi-square analysis for categorical variables. Univariable logistic regression for each postoperative joint complication was used to identify confounding variables amongst the examined demographic variables and comorbidities. Multivariable logistic regression was then used to calculate odds ratio (OR) and corresponding 95\% confidence intervals (C.I.s) for the primary outcomes. Potential confounders adjusted for included sex, CCI, BMI, comorbidities, tobacco use, diabetes mellitus, rheumatoid arthritis, PVD, dementia, liver disease, CHF, CAD, IHD, PHD, CKD, and hypertension.

\section{RESULTS}

\section{STUDY POPULATION}

After exclusion criteria were applied and both cohorts were matched, 2,190 patients were assigned to both the conversion cohort and the THA cohort. By matching patients across gender, there was an equal representation for both cohorts of 740 males and 1,450 females. For BMI, the conversion cohort had a greater percentage of patients with a BMI classification $<30$ (25.30\% vs $21.00 \%$, p <0.001), while the THA cohort had a greater percentage of patients with a BMI classification between $30-40$ (12.88\% vs $15.80 \%$, p 
Table 1. Comparison of gender, BMI, and comorbidities between conversion and THA cohort

\begin{tabular}{|c|c|c|c|}
\hline Demographic Variable & $\begin{array}{c}\text { Conversion Group } \\
\begin{array}{c}(\mathrm{n}=2,190) \\
\mathrm{n}(\%)\end{array}\end{array}$ & $\begin{array}{l}\text { THA Group } \\
(\mathrm{n}=2,190) \\
\mathrm{n}(\%)\end{array}$ & $P$ \\
\hline $\begin{array}{c}\text { Gender, n (\%) } \\
\text { Male } \\
\text { Female }\end{array}$ & $\begin{array}{c}740(33.79) \\
1,450(66.21)\end{array}$ & $\begin{array}{c}740(33.79) \\
1,450(66.21)\end{array}$ & $\begin{array}{l}1.00 \\
1.00\end{array}$ \\
\hline $\begin{array}{c}\text { BMI **, n (\%) } \\
\quad<30 \\
30-40 \\
40+\end{array}$ & $\begin{array}{c}554(25.30) \\
282(12.88) \\
126(5.75)\end{array}$ & $\begin{array}{c}460(21.00) \\
346(15.80) \\
150(6.85)\end{array}$ & $\begin{array}{c}<0.001 \\
<0.001 \\
0.106\end{array}$ \\
\hline $\begin{array}{l}\text { Specific Comorbidities, n (\%) } \\
\text { Tobacco Use } \\
\text { Rheumatoid Arthritis } \\
\text { Liver Disease } \\
\text { CHF } \\
\text { CAD } \\
\text { Pulmonary Heart Disease } \\
\text { Ischemic Heart Disease } \\
\text { COPD } \\
\text { Chronic Kidney Disease } \\
\text { Dementia } \\
\text { Diabetes Mellitus } \\
\text { Hypertension } \\
\text { PVD }\end{array}$ & $\begin{array}{c}478(21.83) \\
164(7.49) \\
148(6.76) \\
359(16.39) \\
757(34.57) \\
740(33.79) \\
164(7.49) \\
694(31.69) \\
363(16.58) \\
70(3.20) \\
786(35.89) \\
1,758(80.27) \\
508(23.20)\end{array}$ & $\begin{array}{c}310(14.16) \\
132(6.03) \\
162(7.40) \\
253(11.55) \\
687(31.37) \\
665(30.37) \\
147(6.71) \\
646(29.50) \\
380(17.35) \\
30(1.37) \\
854(39.00) \\
1,665(76.03) \\
524(23.93)\end{array}$ & $\begin{array}{c}<0.001 \\
0.054 \\
0.409 \\
<0.001 \\
0.024 \\
0.015 \\
0.317 \\
0.116 \\
0.494 \\
<0.001 \\
0.034 \\
<0.001 \\
0.569\end{array}$ \\
\hline
\end{tabular}

THA, Total Hip Arthroplasty; BMI, Body Mass Index; PVD, Peripheral Vascular Disease; CHF, Congestive Heart Failure; CAD, Coronary Artery Disease; IHD, Ischemic Heart Disease; PHD, Pulmonary Heart Disease; CKD, Chronic Kidney Disease; OR, Odds Risk; CI, Confidence Interval

**BMI data was only available for $43.9 \%$ of patients in the conversion cohort and $43.7 \%$ of patients in the THA cohort

$<0.001)$. The conversion cohort contained a larger proportion of patients with tobacco use $(21.83 \%$ vs $14.16 \%$, p $<0.001)$, CHF (16.39\% vs $11.55 \%$, p <0.001), CAD (34.57\% vs $31.37 \%$, $\mathrm{p}<0.001)$, PHD (33.79\% vs $30.37 \%$, $\mathrm{p}<0.001)$, dementia (3.20\% vs $1.37 \%$, p <0.001), diabetes mellitus (35.89\% vs $39.00 \%, \mathrm{p}<0.001)$, and hypertension $(80.27 \%$ vs $76.03 \%, \mathrm{p}<0.001$ ) (Table 1).

\section{MULTIVARIABLE ANALYSIS}

For all joint complications examined at 1 year post-surgery using multivariable analysis, patients in the conversion cohort had significantly increased rates compared to the THA cohort. This included prosthetic joint dislocation $(0.59 \%$ vs 0.18\%: OR 2.95; 95\% CI $1.02-10.65)$, PJI (8.68\% vs $0.73 \%$ : OR 1.38 ; 95\% CI 1.12-1.71), periprosthetic fracture (1.64\% vs $0.50 \%$ : OR 2.95 ; $95 \%$ CI $1.79-4.88)$, aseptic loosening (2.51\% vs $0.18 \%$ : OR 6.86; 95\% CI 4.64-10.23), and revision (1.19\% vs $0.18 \%$ : OR 3.65 ; 95\% CI 2.57-5.20) (Table 2). At 2 years post-surgery, the conversion cohort still exhibited significantly increased rates of joint complications. Prosthetic joint dislocation $(0.73 \%$ vs $0.18 \%$ : OR 3.77 ; $95 \%$ CI 1.36-13.31), PJI (9.63\% vs 0.86\%: OR 2.13 ; $95 \%$ CI $1.22-3.89)$, periprosthetic fracture $(2.32 \%$ vs $0.59 \%$ : OR 3.75; 95\% CI 2.24-6.45), aseptic loosening (2.92\% vs $0.32 \%$ : OR 7.70 ; 95\% CI $5.02-12.18$ ), and revision (1.46\% vs $0.32 \%$ : OR 6.73; 95\% CI 4.58-10.12) were all more likely for patients that underwent conversion THA (Table 2).

\section{DISCUSSION}

With HA and THA being available treatment options for both femoral neck fractures and end-stage hip arthritis, the early failure of HA requiring conversion to THA represents an increased challenge to arthroplasty surgeons. This present study demonstrates that despite controlling for multiple confounding variables, there still exists an increased risk associated with hip conversion to a THA. Patients who underwent conversion had increased risks of prosthetic joint dislocation, PJI, periprosthetic fracture, aseptic loosening, and revision at both 1 and 2 years post-surgery. Each complication examined varied in its risk based on age, gender, BMI, and specific comorbidity. These postoperative complications can be devastating to the patient and surgeon. They also utilize greater resources without commensurate reimbursement, as Sodhi et al. demonstrated less Relative Value Units (RVUs) per minute for conversions compared to elective THA. ${ }^{21}$

One of the highest documented postoperative complications in conversion from a HA to THA is prosthetic dislocations. ${ }^{19,22}$ Sarpong et al. examined 60 patients who underwent conversion from HA to THA and reported a $6.7 \%$ dislocation rate in their conversion group. ${ }^{22}$ Similarly, Sah and Estok demonstrated in a patient cohort of 79 HA to THA conversions over an eleven-year period, there was a $22 \%$ dislocation rate compared to revision THA from a primary THA, which carried an $11 \%$ dislocation rate. ${ }^{19}$ In our study, rates were comparatively higher for the conversion cohort than the THA cohort (1-year: $0.59 \%$ vs. $0.18 \% ; 2$ years: $0.73 \%$ vs. $0.18 \%$ ). 
Table 2. Comparison of complications between conversion and THA cohort

\begin{tabular}{|c|c|c|c|}
\hline Complications & $\begin{array}{l}\text { Conversion Group } \\
(\mathrm{n}=2,190) \\
\mathrm{n}(\%)\end{array}$ & $\begin{array}{c}\text { THA Group } \\
(\mathrm{n}=2,190) \\
\mathrm{n}(\%)\end{array}$ & OR $(95 \% \mathrm{Cl})$ \\
\hline $\begin{array}{l}\text { Prosthetic Joint Dislocation } \\
1 \text { year } \\
2 \text { year }\end{array}$ & $\begin{array}{l}13(0.59) \\
16(0.73)\end{array}$ & $\begin{array}{l}4(0.18) \\
4(0.18)\end{array}$ & $\begin{array}{l}2.95(1.02-10.65) \\
3.77(1.36-13.31)\end{array}$ \\
\hline $\begin{array}{l}\text { Prosthetic Joint Infection } \\
1 \text { year } \\
2 \text { year }\end{array}$ & $\begin{array}{l}190(8.68) \\
211(9.63)\end{array}$ & $\begin{array}{l}16(0.73) \\
19(0.86)\end{array}$ & $\begin{array}{l}1.38(1.12-1.71) \\
2.13(1.22-3.89\end{array}$ \\
\hline $\begin{array}{l}\text { Periprosthetic Fracture } \\
1 \text { year } \\
2 \text { year }\end{array}$ & $\begin{array}{l}36(1.64) \\
51(2.32)\end{array}$ & $\begin{array}{l}11(0.50) \\
13(0.59)\end{array}$ & $\begin{array}{l}2.95(1.79-4.88) \\
3.75(2.24-6.45)\end{array}$ \\
\hline $\begin{array}{l}\text { Aseptic Loosening } \\
1 \text { year } \\
2 \text { year }\end{array}$ & $\begin{array}{l}55(2.51) \\
64(2.92)\end{array}$ & $\begin{array}{l}4(0.18) \\
7(0.32)\end{array}$ & $\begin{array}{l}6.86(4.64-10.23) \\
7.70(5.02-12.18)\end{array}$ \\
\hline $\begin{array}{l}\text { Revision } \\
1 \text { year } \\
2 \text { year }\end{array}$ & $\begin{array}{l}26(1.19) \\
32(1.46)\end{array}$ & $\begin{array}{l}4(0.18) \\
7(0.32)\end{array}$ & $\begin{array}{c}3.65(2.57-5.20) \\
6.73(4.58-10.12)\end{array}$ \\
\hline
\end{tabular}

THA, Total Hip Arthroplasty; OR, Odds Risk; CI, Confidence Interval

A second frequent complication reported after HA conversion to THA is loosening of the prosthetic components, with rates previously reported between $2.3-10 \%$ with a mean follow-up of 6-7 years. ${ }^{23,24}$ In comparison, this study found rates of aseptic loosening to be at $2.51 \% 1$-year postsurgery and $2.92 \% 2$ years post-surgery, both of which were significantly higher than rates of aseptic loosening in the primary THA cohort. These observed rates of loosening can be attributed to a variety of factors such as cementing technique, stem design, and bone resorption rate due to a potential increase in osteoporotic bone in the fragility fracture cohort. ${ }^{23,25}$ While it is not possible with a large database to identify surgical technique or type of stem design used, this study demonstrates that aseptic loosening is a risk factor that arthroplasty surgeons must take into consideration when performing conversion surgery.

Using the clinical practice guidelines from the American Academy of Orthopaedic Surgeons 2019, ${ }^{26}$ to identify risk factor comorbidities associated with PJI, multivariable logistic regression was used to diminish the confounding effect of these comorbidities. Despite the utilization of multivariable logistic regression, rates of PJI were still significantly higher for the conversion cohort compared to the THA cohort at both 1 and 2 years post-surgery (1-year: $8.68 \%$ vs. $0.73 \%$; 2 years: $9.63 \%$ vs. $0.86 \%$ ). In a similar study by Sarpong et al. comparing conversion THA, primary THA, and revision THA, despite not reaching significance, it was found that conversion THA had a $1.7 \%$ PJI rate at 2 years follow-up, in comparison to the $0 \%$ rate found in the primary THA group. ${ }^{12}$ After controlling for multiple variables on the incidence of PJI, this study demonstrates that conversion carries with it an increased risk of PJI. With an increased risk of failure due to revision at both 1 and 2 years following conversion, the conversion cohort demonstrated significantly higher rates of revision in comparison to the primary THA cohort. Further research is warranted to examine the relationship between conversion to THA and PJI.

This study has several limitations. With the utilization of a large database, it is not possible to identify different surgical techniques and stem designs used in the HA to THA conversion surgery. Complications were examined for 1 and 2 year post-surgery time periods; therefore, rates of examined complications cannot necessarily be extrapolated to longer follow-up periods. However, this decision to investigate short to mid-term complications after conversion surgery was due to the majority of complications occurring in the year following initial conversion surgery. In addition, the complexity of medical billing requiring manual input of diagnostic and procedural codes creates the possibility of coding bias. However, these errors are inherent with any database study using administrative claims information. The Center for Medicare and Medicaid (CMS) study demonstrated that such instances make up only $1.0 \%$ of overall payments. ${ }^{27}$ Despite the code CPT-27236 being the selected code of choice for HA for femoral neck fractures, this code does include ORIF for the same indication. Thus a small number of patients in the conversion cohort may have received conversion from ORIF. Finally, despite utilizing multivariable logistic regression to diminish the effect of confounders, the influence of confounders may still exist.

This study is unique in that it utilizes multivariable logistic regression to diminish the effect of multiple confounding variables on rates of postoperative complications in a large sample, thus allowing for confidence in extrapolating the data to the general population when comparing elective THA to conversion THA from HA.

\section{CONCLUSION}

While conversion to a THA is still a frequently performed operation for the failure of HA, this study demonstrates that it carries a higher risk profile for multiple joint complications in short to mid-term follow-up period than primary elective THA. 
All authors declare no conflicts of interest or having received any financial funding for this project. 


\section{REFERENCES}

1. Pivec R, Johnson AJ, Mears SC, Mont MA. Hip arthroplasty. Lancet. 2012;380(9855):1768-1777. doi:1 $\underline{0.1016 / S 0140-6736(12) 60607-2}$

2. Sloan M, Premkumar A, Sheth NP. Projected Volume of Primary Total Joint Arthroplasty in the U.S., 2014 to 2030. J Bone Joint Surg Am. 2018;100(17):1455-1460. doi:10.2106/JBJS.17.01617

3. Charette RS, Sloan M, Lee GC. Not all hip arthroplasties are created equal: increased complications and re-admissions after total hip arthroplasty for femoral neck fractures compared with osteoarthritis. Bone Joint J.

2019;101-B(6_Supple_B):84-90. doi:10.1302/0301-620 X.101B6.BJ-2018-1427.R1

4. Khan AM, Rafferty M, Daurka JS. Hemiarthroplasty compared with total hip arthroplasty in fractured neck of femur: a shift in national practice? Ann $R$ Coll Surg Engl. 2019;101(2):86-92. doi:10.1308/rcsann.201 $\underline{8.0123}$

5. Wu VJ, Ross BJ, Sanchez FL, Billings CR, Sherman WF. Complications Following Total Hip Arthroplasty: A Nationwide Database Study Comparing Elective vs Hip Fracture Cases. J Arthroplasty. Published online March 10, 2020. doi:10.1016/j.arth.2020.03.006

6. Putananon C, Tuchinda H, Arirachakaran A, Wongsak S, Narinsorasak T, Kongtharvonskul J. Comparison of direct anterior, lateral, posterior and posterior-2 approaches in total hip arthroplasty: network meta-analysis. Eur J Orthop Surg Traumatol. 2018;28(2):255-267. doi:10.1007/s00590-017-2046-1

7. Petis S, Howard JL, Lanting BL, Vasarhelyi EM. Surgical approach in primary total hip arthroplasty: anatomy, technique and clinical outcomes. Can J Surg. 2015;58(2):128-139. doi:10.1503/cjs.007214

8. Hansson S, Bulow E, Garland A, Karrholm J, Rogmark C. More hip complications after total hip arthroplasty than after hemi-arthroplasty as hip fracture treatment: analysis of 5,815 matched pairs in the Swedish Hip Arthroplasty Register. Acta Orthop. 2020;91(2):133-138. doi:10.1080/17453674.2019.1690 $\underline{339}$

9. Hongisto MT, Nuotio MS, Luukkaala T, Vaisto O, Pihlajamaki HK. Lateral and Posterior Approaches in Hemiarthroplasty. Scand J Surg. 2018;107(3):260-268. doi:10.1177/1457496917748226
10. Hopley C, Stengel D, Ekkernkamp A, Wich M. Primary total hip arthroplasty versus hemiarthroplasty for displaced intracapsular hip fractures in older patients: systematic review. BMJ. 2010;340:c2332. doi:10.1136/bmj.c2332

11. Liao L, Zhao J, Su W, Ding X, Chen L, Luo S. A meta-analysis of total hip arthroplasty and hemiarthroplasty outcomes for displaced femoral neck fractures. Arch Orthop Trauma Surg. 2012;132(7):1021-1029. doi:10.1007/s00402-012-148 5-8

12. Sarpong NO, Grosso MJ, Lakra A, Held MB, Herndon CL, Cooper HJ. Hemiarthroplasty Conversion: A Comparison to Primary and Revision Total Hip Arthroplasty. J Arthroplasty. 2019;34(6):1168-1173. doi:10.1016/j.arth.2019.02.043

13. Rizkalla JM, Gladnick BP, Bhimani AA, Wood DS, Kitziger KJ, Peters PC Jr. Triaging Total Hip Arthroplasty During the COVID-19 Pandemic. Curr Rev Musculoskelet Med. 2020;13(4):416-424. doi:10.10 07/s12178-020-09642-y

14. Taheriazam A, Saeidinia A. Conversion of failed hemiarthroplasty to total hip arthroplasty: A shortterm follow-up study. Medicine (Baltimore). 2017;96(40):e8235. doi:10.1097/MD.00000000000082 $\underline{35}$

15. Haidukewych GJ, Berry DJ. Hip arthroplasty for salvage of failed treatment of intertrochanteric hip fractures. J Bone Joint Surg Am. 2003;85(5):899-904. d oi:10.2106/00004623-200305000-00019

16. Hernandez NM, Fruth KM, Larson DR, Kremers HM, Sierra RJ. Conversion of Hemiarthroplasty to THA Carries an Increased Risk of Reoperation Compared With Primary and Revision THA. Clin Orthop Relat Res. 2019;477(6):1392-1399. doi:10.109 7/CORR.0000000000000702

17. Hernandez NM, Fruth KM, Larson DR, Kremers HM, Sierra RJ. Conversion of Failed Hemiarthroplasty to Total Hip Arthroplasty Remains High Risk for Subsequent Complications. J Arthroplasty. 2019;34(9):2030-2036. doi:10.1016/j.arth.2019.04.042

18. Schwarzkopf R, Baghoolizadeh M. Conversion total hip arthroplasty: Primary or revision total hip arthroplasty. World J Orthop. 2015;6(10):750-753. do $\mathrm{i}: 10.5312 /$ wjo.v6.i10.750 
19. Sah AP, Estok DM II. Dislocation rate after conversion from hip hemiarthroplasty to total hip arthroplasty. J Bone Joint Surg Am.

2008;90(3):506-516. doi:10.2106/JBIS.G.00479

20. Beck CM, Blair SE, Nana AD. Reimbursement for Hip Fractures: The Impact of Varied Current Procedural Terminology Coding Using Relative Value Units. J Arthroplasty. 2020;35(12):3464-3466. doi:10.1 016/j.arth.2020.06.088

21. Sodhi N, Dalton SE, Garbarino LJ, et al. Not all primary total hip arthroplasties are equal-so is there a difference in reimbursement? Ann Transl Med. 2019;7(4):74. doi:10.21037/atm.2018.08.14

22. Sarpong NO, Grosso MJ, Lakra A, et al. Outcomes and survivorship after conversion of failed hip hemiarthroplasty to total hip arthroplasty. Hip Int. Published online 2019:1120700019891427. doi:10.117 7/1120700019891427

23. Pankaj A, Malhotra R, Bhan S. Conversion of failed hemiarthroplasty to total hip arthroplasty: a short to mid-term follow-up study. Indian J Orthop. 2008;42(3):294-300. doi:10.4103/0019-5413.41852
24. Sierra RJ, Cabanela ME. Conversion of failed hip hemiarthroplasties after femoral neck fractures. Clin Orthop Relat Res. 2002;(399):129-139. doi:10.1097/00 003086-200206000-00015

25. Hammad A, Abdel-Aal A. Conversion total hip arthroplasty: functional outcome in Egyptian population. Acta Orthop Belg. 2006;72(5):549-554.

26. American Academy of Orthopedic Surgeons. Clinical practice guideline and evidence report on the diagnosis and prevention of periprosthetic joint infection. Published 2019. Accessed August 13, 2020. https://aaos.org/quality/quality-programs/tumor-infe ction-and-military-medicine-programs/diagnosis--pr evention-of-periprosthetic-joint-infections/

27. Centers for Medicare and Medicaid Services. 2019 Medicare Fee-for-Service Supplemental Improper Payment Data. Published 2019. Accessed April 2021. https://www.cms.gov/research-statistics-data-and-sy stemsmonitoring-programsmedicare-ffs-complianceprogramscertcert/2019-medicare-fee-service-supple mental-improper-payment-data 


\section{SUPPLEMENTARY MATERIALS}

\section{Appendix Table A1}

Download: https://orthopedicreviews.openmedicalpublishing.org/article/25539-complication-rates-ofhemiarthroplasty-conversion-to-total-hip-arthroplasty-versus-primary-total-hip-arthroplasty/attachment/ 65538.docx 